\section{Comparison of techniques for distinguishing staphylococci and micrococci}

LUCY BOSSCHER-ZONDERMAN AND INGRID SMITH From the Hospital Epidemiology Unit, University Hospital, Leiden, The Netherlands

In the past a variety of tests have been proposed for the differentiation of the genus Staphylococcus from Micrococcus. The most fundamental division between these two genera of Gram-positive cocci is in the DNA base composition: staphylococci have a guanine and cytosine (GC) content of about 30 to $40 \%$ and micrococci of about 50 to $75 \%$ (Kocur, Bergan, and Mortensen, 1971). The determination of the percentage GC content is, however, too tedious and time consuming for epidemiological work involving many specimens. Other methods used have consisted mainly of demonstrations of the ability of Staphylococcus species to grow and ferment glucose anaerobically; Micrococcus species do not have this ability; they are oxidative.

The principal taxonomic scheme for the Micrococcaceae is that of Baird-Parker $(1963,1965)$; this scheme uses the ability of Staphylococcus species to ferment glucose anaerobically and has been the basis of epidemiological studies in this department. During the course of these studies other methods, principally the thioglycollate medium of Evans and Kloos (1972) and the novobiocin sensitivity method

Received for publication 5 February 1973. of Jeffries (1968), were also used. This paper reports a comparison of these methods.

\section{Materials and Methods}

Strains of Gram-positive, catalase-positive, coagulase-negative cocci isolated during epidemiological studies were used. Staphylococcus aureus phage propagating strains were used as control 'positive' organisms and Sarcina (tetrad forming, nonfermentative, aerobic cocci with a typical colony and yellow pigment) were used as 'negative' controls. Anaerobic glucose medium was prepared according to Baird-Parker (1963), thioglycollate according to Evans and Kloos (1972), and novobiocin sensitivity tests were carried out according to Jeffries' disc method on DST agar (Oxoid).

\section{Results}

The results are shown in table I. It can be seen that novobiocin was of no value in this series of strains; no explanation can be found. There was good agreement between the other two methods for strains classified as staphylococci (SII-SVI) by BairdParker's technique but not for those classified as micrococci (M1-M7). The distribution of strains from various surgical lesions was changed by substituting the results obtained from the thioglycollate medium of Evans and Kloos for the anaerobic glucose medium of Baird-Parker; the percentage of M3 strains was reduced but the differences are not statistically different (table II). M3 strains were obtained significantly more frequently from sites below the waist when grouped according to BairdParker $\left(\mathrm{P}<1 \%, \chi^{2}=10.3\right)$ but not when grouped according to Evans and Kloos ( $>>5 \%)$.

\begin{tabular}{|c|c|c|c|c|c|}
\hline \multirow{2}{*}{$\begin{array}{l}\text { Results According to } \\
\text { Baird-Parker Scheme }\end{array}$} & \multicolumn{2}{|c|}{ Anaerobic Growth (Evans and Kloos, 1972) } & \multicolumn{2}{|c|}{ Novobiocin ${ }^{2}$} & \multirow[t]{2}{*}{ Total Strains Tested } \\
\hline & Staphylococcus & Micrococcus & Sensitive & Resistant & \\
\hline Staph. aureus & 23 & $\mathbf{0}$ & 22 & 1 & 23 \\
\hline SII & 123 & 2 & 115 & 10 & 125 \\
\hline SIII & 9 & 0 & 7 & 2 & 9 \\
\hline SIV & 1 & $\mathbf{0}$ & 1 & 0 & 1 \\
\hline SV & 14 & 0 & 14 & 0 & 14 \\
\hline SVI & 30 & 4 & 34 & 0 & 34 \\
\hline M1 & 14 & 1 & 15 & 0 & 15 \\
\hline M2 & 16 & 5 & 21 & 0 & 21 \\
\hline M3 & 11 & 19 & 29 & 1 & 30 \\
\hline M4 & 0 & 1 & 1 & $\mathbf{0}$ & 1 \\
\hline M5 & 5 & 4 & 8 & 1 & 9 \\
\hline M6 & $\mathbf{0}$ & 1 & $\mathbf{0}$ & 1 & 1 \\
\hline Sarcina & $\mathbf{0}$ & 18 & 17 & 1 & 18 \\
\hline
\end{tabular}

Table I Distinguishing staphylococci from micrococci

${ }^{1}$ Jeffries (1968) found that staphylococci and M7 were sensitive whilst micrococcus species M1 to M6 were resistant. $\mathbf{S}=$ Staphylococcus $\quad \mathbf{M}=$ Micrococcus 


\begin{tabular}{|c|c|c|c|c|c|c|c|c|}
\hline \multirow[t]{4}{*}{ Organism } & \multicolumn{8}{|c|}{ Percentage Distribution by Site } \\
\hline & \multicolumn{2}{|l|}{ Abdomen } & \multicolumn{2}{|l|}{ Leg } & \multicolumn{2}{|l|}{ Perineum } & \multicolumn{2}{|l|}{ Other } \\
\hline & \multicolumn{8}{|c|}{ Anaerobic Glucose Medium } \\
\hline & Baird-Parker ${ }^{1}$ & Evans and Kloos & Baird-Parker & Evans and Kloos & Baird-Parker & Evans and Kloos & Baird-Parker & Evans and Kloos \\
\hline SII & 53 & 57 & 34 & 41 & 42 & 48 & 51 & 56 \\
\hline SIII & 4 & 4 & 3 & 3 & 2 & 2 & 4 & 4 \\
\hline SIV & 1 & 4 & 0 & 9 & 0 & 0 & 0 & 11 \\
\hline SV & 3 & 3 & 6 & 3 & 6 & 4 & 7 & 9 \\
\hline SVI & 17 & 16 & 6 & 22 & 20 & 28 & 7 & 11 \\
\hline M1 & 5 & 0 & 9 & 0 & 4 & 2 & 6 & 0 \\
\hline M2 & 6 & 3 & 9 & 3 & 4 & 0 & 12 & 2 \\
\hline M3 & 7 & 10 & 22 & 13 & 20 & 10 & 7 & 6 \\
\hline M4 & 1 & 1 & 0 & 0 & 0 & 4 & 0 & $\mathbf{0}$ \\
\hline M5 & 1 & 1 & 9 & 6 & 0 & 0 & 6 & 0 \\
\hline M6 & 0 & 0 & 0 & 0 & 0 & 0 & 1 & 1 \\
\hline Total strains & 94 & & 32 & & 49 & & 85 & \\
\hline
\end{tabular}

Table II Distribution of strains according to sites of lesions

${ }^{1}$ Baird-Parker = Anaerobic glucose according to Baird-Parker classification.

${ }^{2}$ Evans and Kloos = Anaerobic glucose (thioglycollate) medium of Evans and Kloos.

$\mathbf{S}=$ Staphylococcus

$\mathbf{M}=$ Micrococcus

\section{Comment}

Evans and Kloos reported their thioglycollate medium to give results which more closely approached the percentage GC content than did others. In the present series the Staphylococcus aureus and Sarcina sp gave results according to expectation. St. epidermidis strains (Baird-Parker's SII, III, IV, V, VI) gave agreement in all except six $(3 \%)$ strains. The equivocal groups were those identified as Micrococcus saprophyticus (M1, 2, and 3) and M. lactis (M5 and 6) according to BairdParker, for over $60 \%$ of these strains were Staphylococcus species using the medium of Evans and Kloos (1972). This is not surprising for it has long been known that such strains, whilst lacking or having a poor ability to ferment glucose anaerobically, may nevertheless have a low GC value (Silvestri and Hill, 1965; Auletta and Kennedy, 1966). This survey has identified S II and S VI as the most common strains in wounds with $\mathbf{M} 3$ strains common in lesions of the legs and perineum but not of the abdomen or other sites. This is in agreement with the studies of others. Holt (1969) found S II to be the organism responsible for infection of ventriculoatrial shunts in babies with hydrocephalus. Coagulase-negative staphylococci isolated from blood stream infections are also of this type (Rames, Wise, Goodman, and Piel, 1970). Urinary tract infection in patients outside the hospital environment is most frequently with type M 3
(Mitchell, 1968). Few other studies have been reported of complete characterization of cocci from surgical lesions.

These strains were collected during studies on the epidemiology of wound infection. We are grateful to the members of the Infection Committee for permission to publish this material, to $\mathrm{Mr} \mathrm{M}$. $\mathrm{H}$. Rebel and Sister G. Berbee who collected specimens, and to Dr W. C. Noble for help and advice.

\section{References}

Auletta, A. E., and Kennedy, E. R. (1966). Deoxyribonucleic acid base composition of some members of the Micrococcaceae. J. Bact., 92, 28-34.

Baird-Parker, A. C. (1963). A classification of micrococci and staphylococci based on physiological and biochemical tests. J. gen. Microbiol., 30, 409-427.

Baird-Parker, A. C. (1965). The classification of staphylococci and micrococci from world-wide sources. J. gen. Microbiol., 38, 363-387.

Evans, J. B., and Kloos, W. E. (1972). Use of shake cultures in a semisolid thioglycolate medium for differentiating staphylococci from micrococci. Appl. Microbiol., 23, 326-331.

Holt, R. J. (1969). The classification of staphylococci from colonized ventriculo-atrial shunts. J. clin. Path., 22, 475-482.

Jeffries, L. (1968). Sensitivity to novobiocin and lysozyme in the classification of micrococcaceae. J. appl. Bact., 31, 436-442.

Kocur, M., Bergan, T., and Mortensen, N. (1971). DNA base composition of gram-positive cocci, J. gen. Microbiol, 69, 167-183.

Mitchell, R. G. (1968). Classification of Staphylococcus albus strains isolated from the urinary tract. J. clin. Path., 21, 93-96.

Rames, L. Wise, B., Goodman, J. R., and Piel. C. F. (1970). Renal disease with Staphylococcus albus bacteremia; a complication in ventriculoatrial shunts. J. Amer. med. Ass., 212, 1671-1677.

Silvestri, L. G., and Hill, L. R. (1965). Agreement between deoxyribonucleic acid base composition and taxometric classification of Gram-positive cocci. J. Bact., 90, 136-140. 